\title{
Iliac artery injury during trocar entry in laparoscopic surgery
}

\author{
Mehmet Can Aydın, @ Cihan Gökler, @ Kuntay Kaplan, @ Cüneyt Kayaalp
}

Department of Gastroenterology Surgery, İnönü University Faculty of Medicine, Malatya, Turkey

To the Editor,

In the diagnosis and surgical treatment of benign and malign diseases in general surgery, gynecology and urology clinics, laparoscopy is one of the preferred surgical method with less pain, less surgical site infections, earlier mobilization and less hospital stay. Yet, this method has some serious complications, major- minor vascular injuries, bladder and bowel injuries are the most commons.

Four months ago a male patient, 76 years old, with BMI: 23 had diagnostic laparoscopic surgery for gastric cancer. Diagnostic laparoscopy was done with trocar entry point from $3 \mathrm{~cm}$ left lateral to the umbilicus. When a locally advanced tumor was detected in laparoscopy, the patient was planned to neoadjuvant chemotherapy. After a 4 months period of neoadjuvant chemotherapy,the patient was re-evulated for a second operation. After entering with Veres needle from left upper quadrant to the abdomen and providing pneumoperitoneum, a $12 \mathrm{~mm}$ trocar (Covidien) was inserted from $3 \mathrm{~cm}$ lateral of the umbilicus (previous trocar-site). Shortly after the trocar entry, a hemoperitoneum was observed, hypotension and tachycardia developed. Despite detailed laparoscopic exploration, bleeding origin couldn't be found and urgent laparotomy applied for the suspicion of major vascular injury. During laparotomy, a retroperitoneal hematoma, compatible with trocar entry and approximately $700 \mathrm{cc}$ intra-abdominal hemorrhage were observed. Despite ongoing bleed- ing the origin couldn't be detected. Retroperitoneum was opened, hematoma was cleaned, distal aorta and iliac arteries were explored. There was an active arterial bleeding from a $5 \mathrm{~mm}$ in size left iliac artery injury $2 \mathrm{~cm}$ below the aortic bifurcation. Hemorrhage was controlled with proximal and distally placed vascular clamps, injured site was primarily repaired with 5.0 prolene sutures one by one and the patient was heparinized (Fig. 1). Still ongoing hemorrhage from minor full-thickness injury of meso-ileum was controlled by sutures. Following hemostasis, it was observed that the gastric cardia tumor was invading the aorta and extending to distal esophagus. (There was no finding of aortic invasion in the pre-operative computed tomography.) Operation was terminated by accepting that the tumor was unresectable. Total bleeding amount was $1000 \mathrm{cc}$. The patient was taken to intensive care. Hemodynamics and hemogram values were stable during two days in intensive care unit. On post-operative second day, abdominal drain was removed. Doppler ultrasound revealed that bilateral iliac artery and lower extremity arteries and veins were patent. The patient was discharged on the 4th post operative day without any problem.

Major vascular injuries are quite rare (0.05-0.1\%) during laparoscopy and when it occurs, it causes significant mortality and morbidity ${ }^{[1,2]}$ They often occur during Veres needle or trocar entry to the abdomen. ${ }^{[3,4]}$ This risk increases especially in slim patients. ${ }^{[5]}$ The most frequently injured 


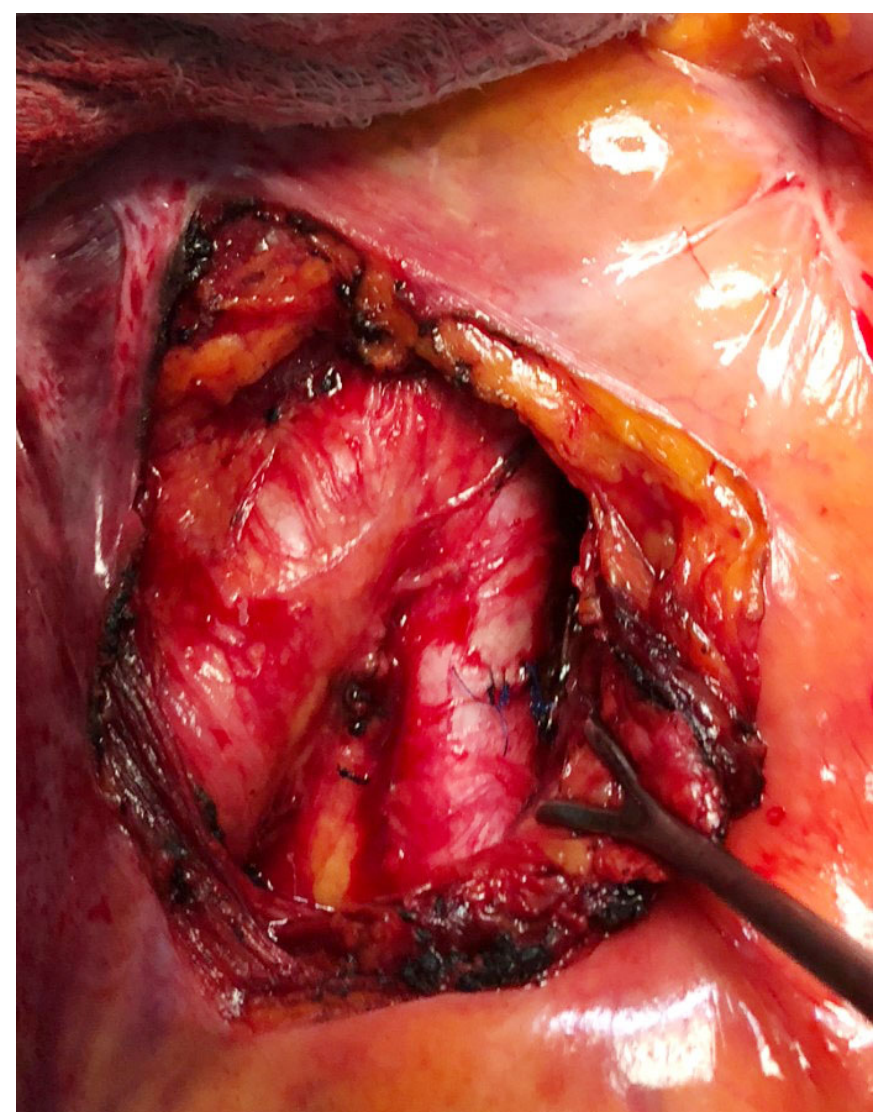

Figure 1. Exploration of the retroperitoneal area and appearance of the left iliac artery injury line after primary closure.

vessels are the abdominal aorta, iliac arteries and vena cava. There is still no accepted consensus for the safest trocar entry ${ }^{[6]}$ In the literature, most related studies are from gynecology and urology clinics and rarely from general surgery departments. Especially in patients who are slim and have previous abdominal surgery, it may be safer to make trocar entries in a different site because of possible intra-abdominal adhesions. If the bleeding source can be demonstrated in laparoscopic exploration, bleeding control and vessel repair has been reported by laparoscopy. ${ }^{[7]}$ We consider that laparotomy should be performed in case of sudden hypotension, tachycardia or hemorrhage.
The trocar entry route should be examined first and then the injured vessels should be explored. The patient should be followed up with administration of anticoagulant in the intensive care conditions in the postoperative period by Doppler ultrasonography controls. We accepted the major vascular injuries as the injuries of aorta, vena cava and iliac vassels. In our center major laparoscopic vascular injury was seen only in a patient among approximately 1500 laparoscopic operations between 2009-2018 and our incidence was $0.07 \%$ and this rate is compatible with the literature. However, there were more minor vascular injuries in four patients regarding with meso-ileum and laparotomy was performed in all these patients. No mortality observed in any of five patients.

\section{References}

1. Fuller J, Scott W, Ashar B, Corrado J. Laparoscopic Trocar Injuries: A Report From a US Food and Drug Administration Centre For Devices and Radiological Health Systematic Technology Assessment of Medical Products Committee. Washington, DC: FDA; 2005.

2. Mechchat A, Bagan P. Management of major vascular complications of laparoscopic surgery. J Visc Surg 2010;147:14553. [CrossRef]

3. Pickett SD, Rodewald KJ, Billow MR, Giannios NM, Hurd WW. Avoiding major vessel injury during laparoscopic instrument insertion. Obstet Gynecol Clin North Am 2010;37:387-97.

4. Dunne N, Booth MI, Dehn TC. Establishing pneumoperitoneum: Verres or Hasson? The debate continues. Ann R Coll Surg Engl 2011;93:22-4. [CrossRef]

5. Afifi Y, Raza A, Balogun M, Khan KS, Holders R. New nomogram for safe laparoscopic entry to reduce vascular injury. J Obstet Gynaecol 2011;31:69-72. [CrossRef]

6. Tinelli A, Malvasi A, Istre O, Keckstein J, Stark M, Mettler L. Abdominal access in gynaecological laparoscopy: a comparison between direct optical and blind closed access by Verres needle. Eur J Obstet Gynecol Reprod Biol 2010;148:191-4.

7. Jafari MD, Pigazzi A. Techniques for laparoscopic repair of major intraoperative vascular injury: case reports and review of literature. Surg Endosc 2013;27:3021-7. [CrossRef] 